\title{
From eosinophilic esophagitis to esophagus perforation: clinical management strategies
}

\author{
Tabita Timeea Scutaru*,1, Péter Kupcsulik ${ }^{1}$, Péter Sahin ${ }^{2}$, Ákos Szücs ${ }^{1}$ \\ ${ }^{1}$ Semmelweis University's First Department of Surgery, Budapest, Hungary; ${ }^{2} J a h n$ Ferenc South-Pest \\ Hospital and Clinic, Budapest, Hungary
}

\begin{abstract}
Introduction: Eosinophilic esophagitis is a chronic, antigen-mediated inflammation of the esophagus. The disease is most common at young ages, with a male to female ratio of 3:1. Eosinophilic granulocyte infiltration induced by oral/aeroantigens in the esophagus, mucosal hyperplasia, and fibrosis of the subepithelial layers can lead to constriction, dysphagia, blockage and esophageal perforation. Case report: A 36-year-old male patient presented in June 2016 with dysphagia as the main complaint. Workup with plain chest radiography with a water soluble contrast swallow did not reveal any pathological lesions. The patient's swallowing difficulties persisted and one year later he was treated by esophageal food bolus impaction (EFBI) in another institution. A new plain chest radiography with a water soluble contrast swallow confirmed a $9 \mathrm{~cm}$ long stricture in the middle third with an EFBI. During gastroscopy, a clinical picture of eosinophilic esophagitis was noted, with partially destroyed foreign body at $25 \mathrm{~cm}$ and iatrogenic perforation at the upper half of the esophagus. After preoperative intensive care unit valuation and preparation, transhiatal esophagectomy without thoracotomy and cervical esophagostomy was performed with pyloromyotomy and feeding jejunostomy. The postoperative period was uneventful. Histological examination confirmed the presence of strictures and perforation on the background of eosinophilic esophagitis. Elective esophageal reconstruction with cervical esophagogastric anastomosis was performed on January 2018. Control blood tests revealed persistent eosinophilia, while the plain chest radiography with a water soluble contrast swallow showed no contrast leakage. Per os nutrition was resumed and the patient was discharged in good general condition. Conclusions: Eosinophilic esophagitis is a rare and difficult to diagnose entity due to its non-specific clinical presentation. In order to avoid complications and undesired delay in diagnosis, one should take into consideration this entity in every clinical situation of a young male patient with swallowing complaints.
\end{abstract}

Keywords: eosinophilic esophagitis; dysphagia; esophageal food bolus impaction; esophageal perforation

\section{Introduction}

Eosinophilic esophagitis (EoE) represents a chronic, local immune-mediated inflammation of the esophagus. Oral and/or airborne allergens can induce eosinophil granulocyte infiltration, mucosal hyperplasia,

Received: May 2019; Accepted after review: June 2019; Published: June 2019.

${ }^{*}$ Corresponding author: Tabita Timeea Scutaru, First Department of Surgery Semmelweis University, Üllői út 78, 1082 Budapest, Hungary

Email: tabiscutaru@gmail.com and fibrosis of the subepithelial layers of the esophagus [1, 2]. EoE has received special attention over the past 20 years as a standalone disease. While it is most common in childhood or in young adulthood, 28.6 to $85 \%$ of patients have a history of allergic rhinitis, sinusitis, asthma or atopic dermatitis [3, 4].

EoE patients can show a wide range of symptoms depending on the age of onset. In children with ages between 2 to 12 years, gastro-esophageal reflux disease (GERD) symptoms (nausea, vomiting, abdominal pain, heartburn, regurgitation) that do not respond to medication are most common. In adults, the 
most common symptom of EoE is periodic dysphagia and esophageal food bolus impaction (EFBI). Spontaneous esophageal perforation may be a serious complication of EoE $[2,5]$.

The main diagnostic methods of EoE are endoscopy (small, whitish, dotted or linear exudates, mucosal edema, mucous membranes, fine concentric mucosal rings corrugated esophagus, crepe paper, pavement, long-section stricture, scars), histology (the number of eosinophil granulocytes of the esophageal tissue exceeds 15 eosinophil granulocyte/highmagnitude field of vision and biopsy samples from other parts of the digestive tract do not shows any significant difference), as well as allergic tests (high serum $\mathrm{lgE}$ level), $\mathrm{pH}$ monitoring (according to some studies, the presence of GERD may facilitate the formation of EoE), endoscopic ultrasound, manometric examinations and plain chest radiography with a water soluble contrast swallow [2, 4].

In the treatment of EoE, diet (remove trigger antigens from the diet) and antiinflammatory drugs (PPI therapy, systemic or topical corticosteroid treatment - budesonide, fluticasone) are playing important role and in case of severe constriction mechanical dilation is the solution. The mechanical dilatation of esophagus may cause excessive chest pain following the intervention, esophageal bleeding and perforation [5-7].

\section{Case report}

We present the case of a 36-year-old male with no pathological history of interest. In June 2016 the patient was admitted to the outpatient clinic with swallowing difficulties of 3 days of evolution. The plain chest radiography with a water soluble contrast swallow did not revealed any pathological lesions and PPI therapy (Pantoprazol 2x20mg/day, 12 weeks) in combination with six food elimination diet (milk products, eggs, wheat, soy, peanut/tree nuts, and fish/shellfish) was administered. Despite the treatment, the patient's swallowing complaints persisted, but the young male was noncompliant for follow-up.

One year later, the patient presented to the emergency department with swallowing complaints and EFBI. Plain chest radiography with a water soluble contrast swallow revealed a $9 \mathrm{~cm}$ long stricture and food bolus impaction in the middle third of the esophagus (Figure 1). In order to evacuate the foreign body from the esophagus gastroscopy was executed. Gastroscopy examination described the food bolus impaction at $25 \mathrm{~cm}$ from dental arch and specific macroscopic image of eosinophilic esophagitis (Figure 2).

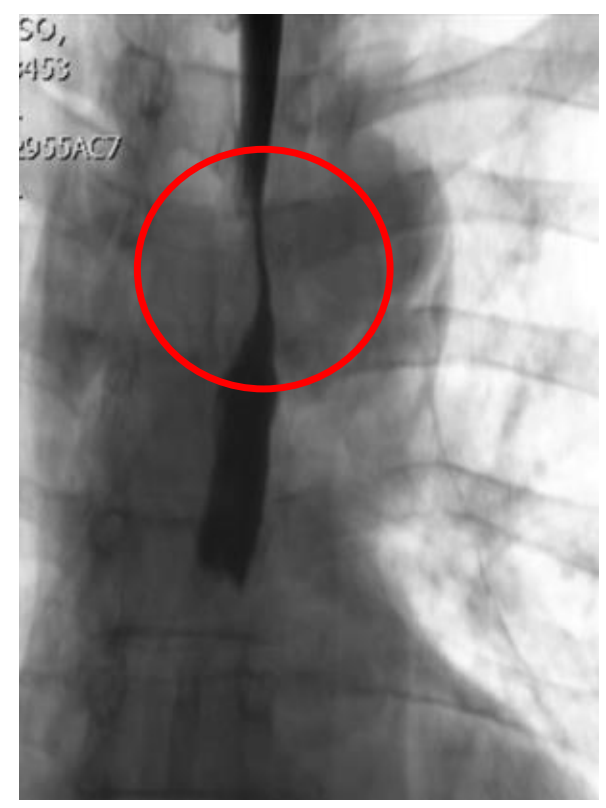

Fig. 1. Plain chest radiography with a water soluble contrast swallow: $9 \mathrm{~cm}$ long stricture and food bolus impaction in the middle third of the esophagus 


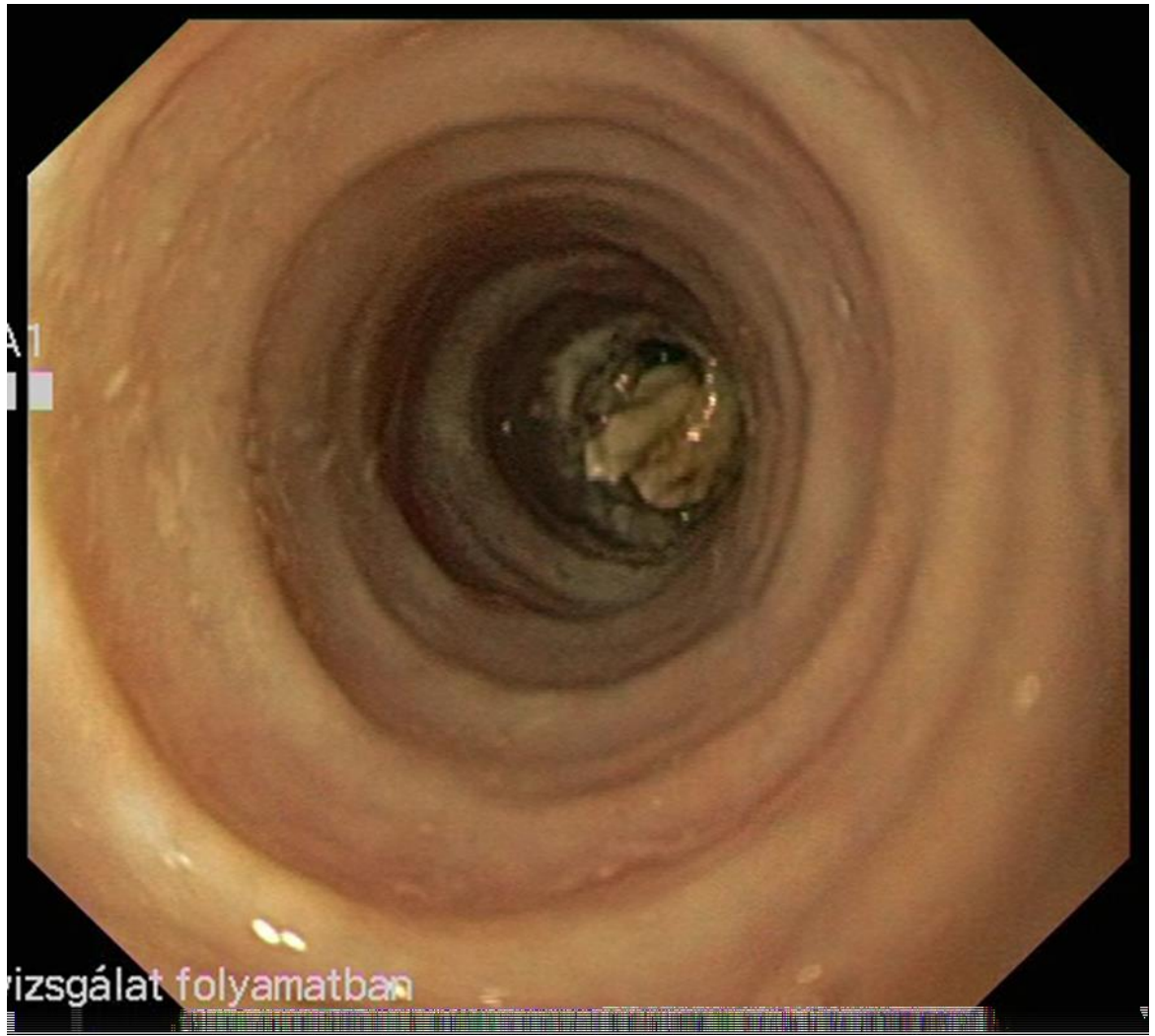

Fig. 2. Endoscopic aspect of EoE (small, whitish, dotted exudates, ring formation, crepe paper, pavement, longsection stricture)

Subcutaneous emphysema was detected while removing the foreign body. Due to high risk of complications, a thoracic computer tomography was made confirming the suspicion of pneumomediastinum (Figure 3 ).

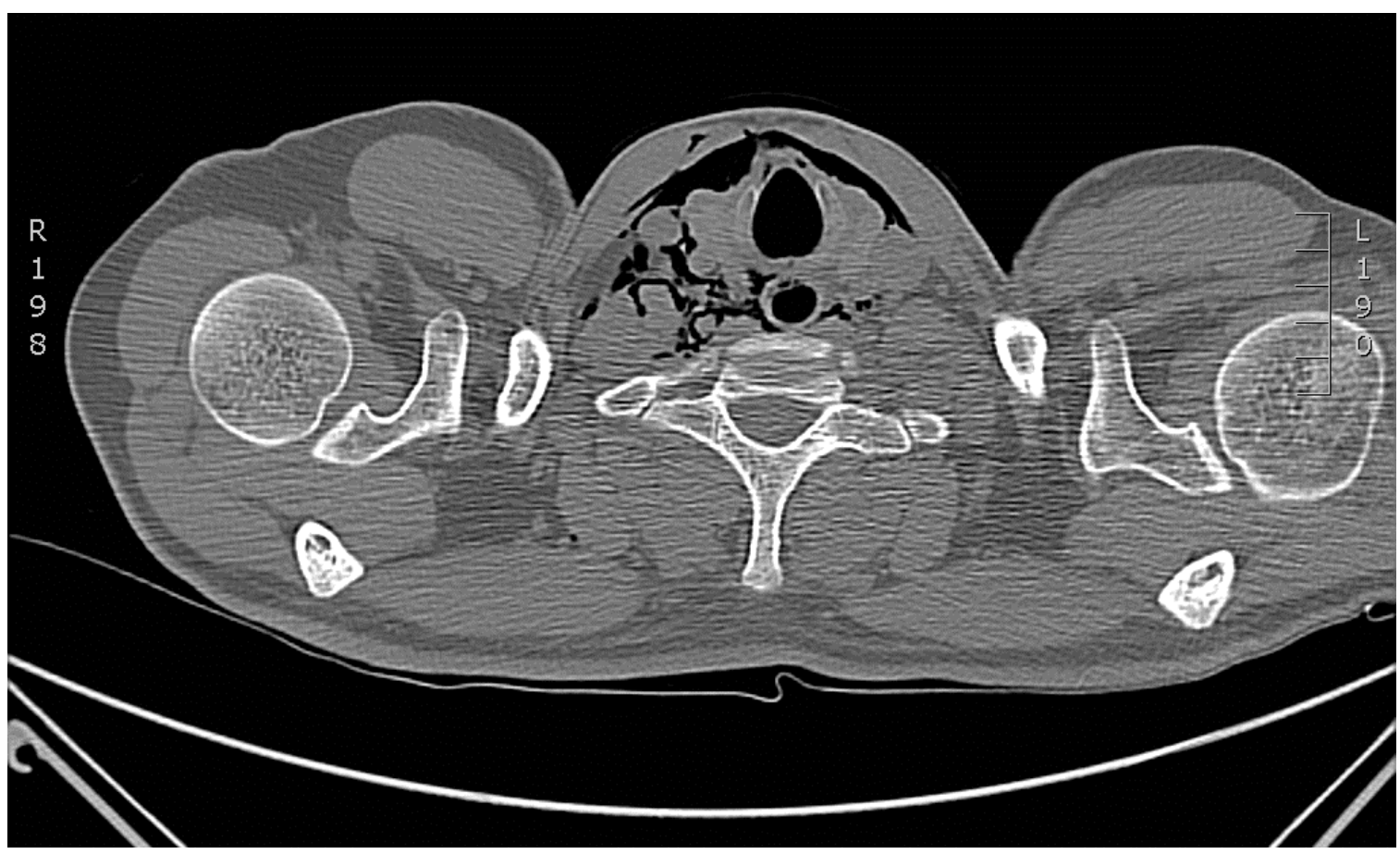

Fig. 3. Thoracic CT: aspect of pneumomediastinum and presence of subcutaneous emphysema 
A new upper Gl endoscopy described esophageal perforation (Figure 4). Thereby, despite the attempts to close the esophageal perforation with 3 clips, the patient's general condition declined and was urgently transferred to our surgical department with the diagnosis of esophageal perforation.

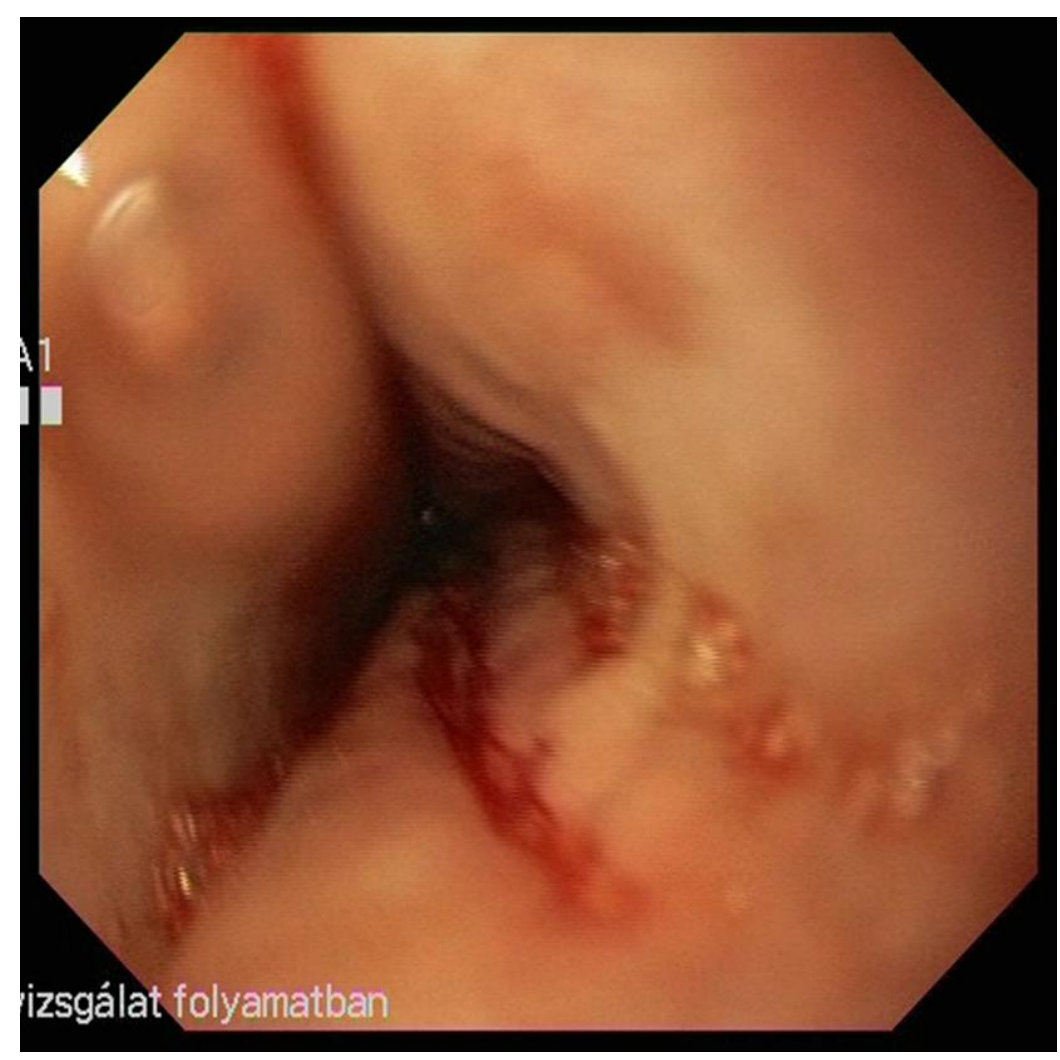

Fig. 4. Endoscopic aspect of esophageal perforation

On admission, the physical examination shows a febrile patient $\left(38.2^{\circ} \mathrm{C}\right)$ with continuous chest pain, that increases with motion, no emphysema, auscultatory bilateral soft rough breathing sounds on a hemodynamically stable patient (blood pressure $140 / 90 \mathrm{mmHg}$, arterial pulse $85 \mathrm{bpm}$, oxygen saturation 98\%). Blood tests (Table 1) showed increased inflammatory markers, including mild eosinophilia, which sustained EoE. Plain chest radiography with a water soluble contrast swallow was performed in order to confirm the diagnosis of esophageal perforation (Figure 5).

Table 1. Blood test on admission

\begin{tabular}{llll} 
Test Name & Result & Unit & Normal Value \\
\hline Lymphocytes & 3.6 & $\%$ & $25-45$ \\
\hline NEUABS & 22.9 & Giga/L & $1.8-7$ \\
INR & 1.05 & INR & 1.1 \\
\hline Total Bilirubin & 21.8 & umol/L & 17 \\
Direct & 37 & umol/L & $1.71-20.5$ \\
Bilirubin & 20 & $\mathrm{U} / \mathrm{L}$ & $40-50$ \\
TGO & 16 & $\mathrm{U} / \mathrm{L}$ & $40-50$ \\
TGP & 14 & $\mathrm{U} / \mathrm{L}$ & $50-70$ \\
GGT & 3.2 & $\mathrm{mmol} / \mathrm{L}$ & $3.5-5$ \\
K & 0.54 & $\mathrm{ug} / \mathrm{L}$ & $<0.5$ \\
Procalcitonin & 27.3 & $\mathrm{mg} / \mathrm{L}$ & $<5$ \\
CRP & 2.5 &
\end{tabular}




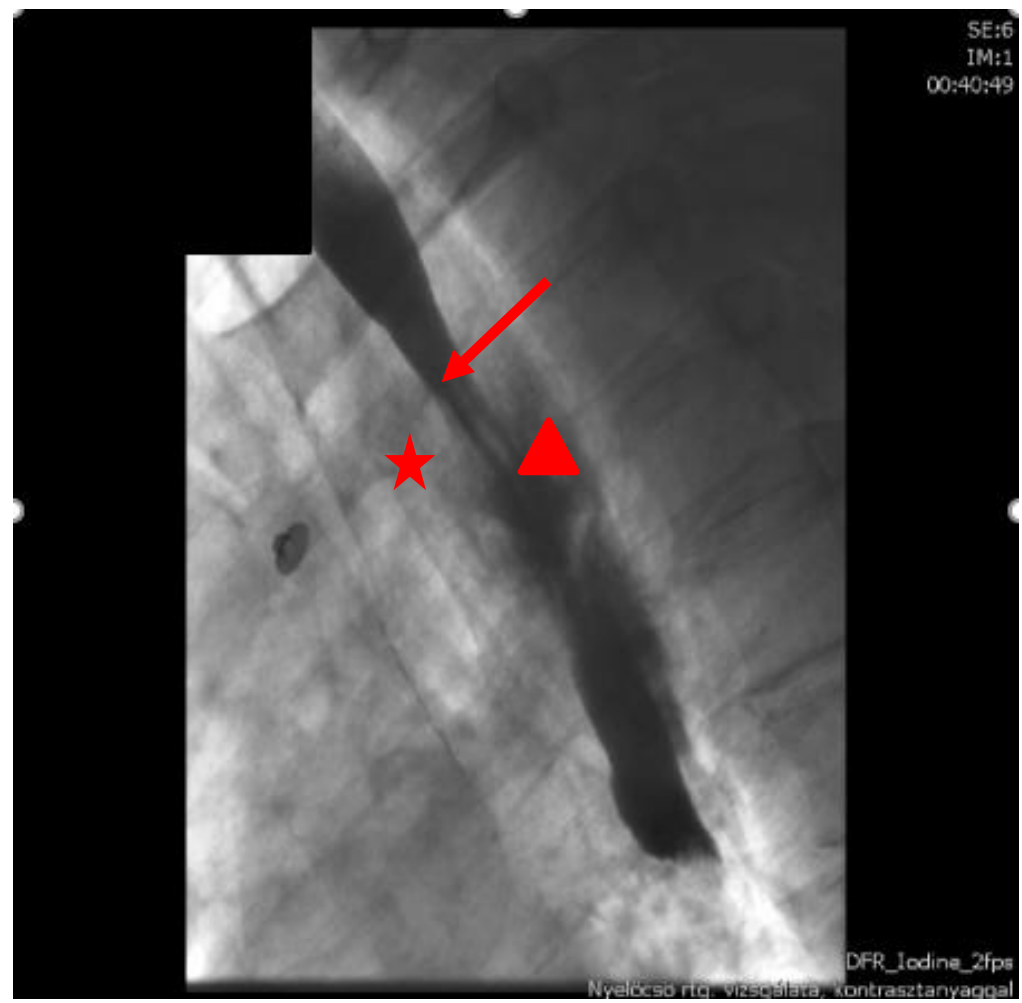

Fig. 5. Plain chest radiography with a water soluble contrast swallow: esophageal stricture (arrow), esophageal food bolus impaction (arrow head), esophageal perforation (star)

After preoperative intensive care unit (ICU) evaluation and preparation, transhiatal esophagectomy (THE) and cervical esophagostomy were performed with pyloromyotomy and feeding jejunostomy. The diagnosis of eosinophilic esophagitis was confirmed by histologic examination (Figures $6 a$ and $6 b)$.

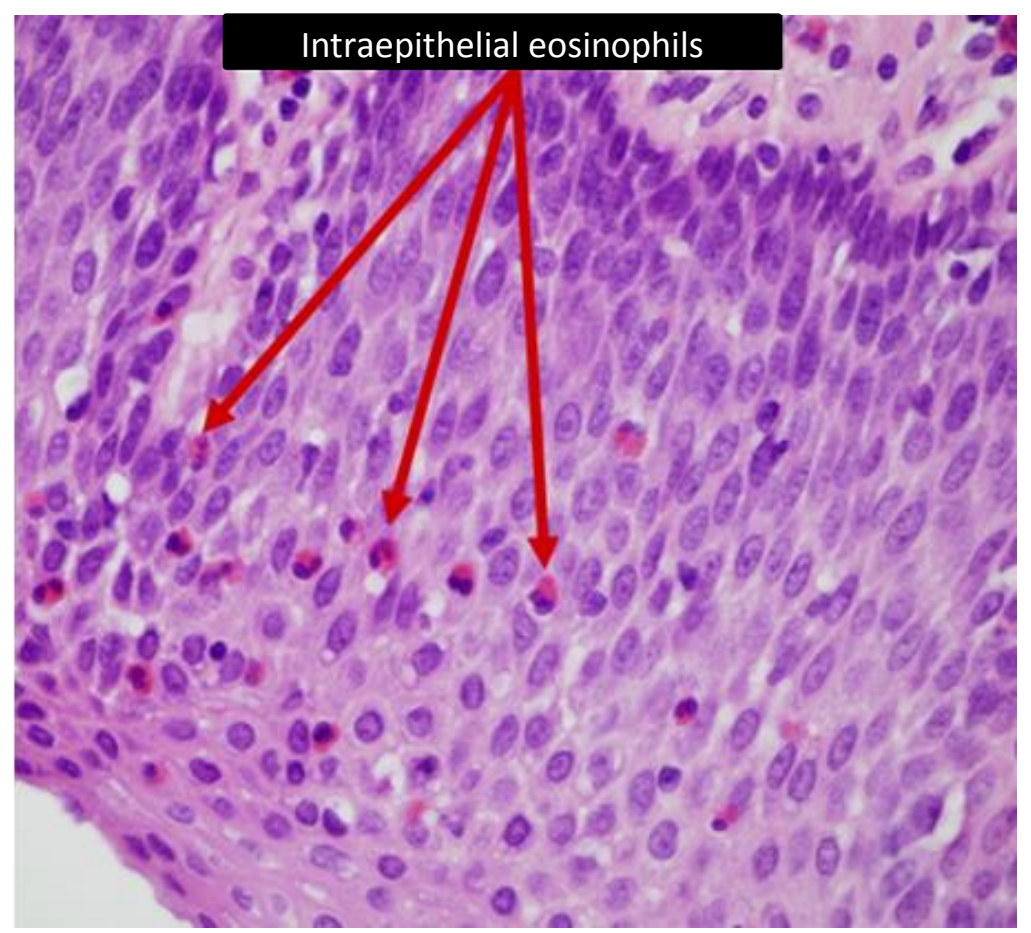

Fig. 6a. Eosinophilic esophagitis: thickened basal layer, numerous intraepithelial eosinophils (red arrows), dilated intercellular spaces (spongiosis) (HE, x60) 


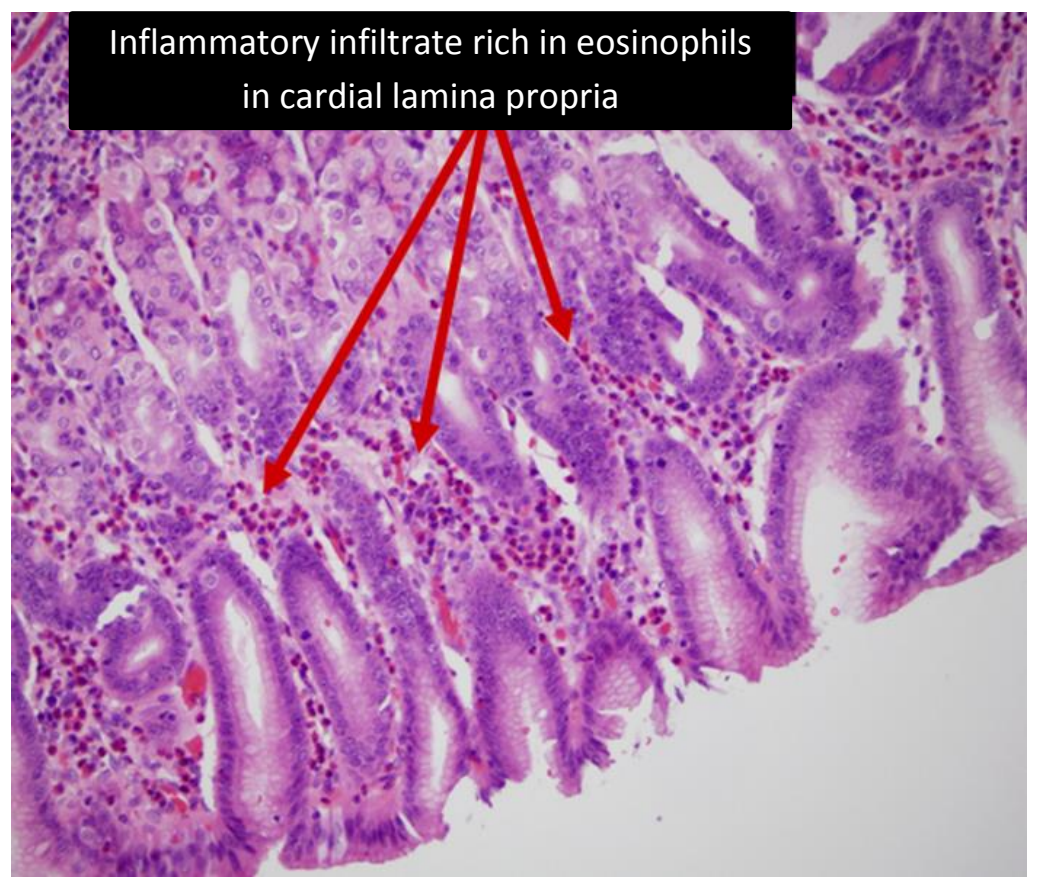

Fig. 6b. Eosinophilic gastritis: $>25$ eosinophils/high power field in cardial mucosa and congestion (HE, x20)

In the postoperative period, we focused on patient nutritional support, imagistic control (Figures $7 \mathrm{a}$ and $7 \mathrm{~b}$ ) and blood tests (Table 2) survey, as follows. Considering that the maintenance of a good nutritional status of the patient is a crucial step, he received the nutritional support according to existing protocols [8, 9]. On postoperative day 1 a control contrast study was done in order to confirm the correct position of the jejunostomy tube, then $500 \mathrm{ml}$ of tea (dextrose solution) and $500 \mathrm{ml}$ of Nutrison Advanced Peptisorb (ingredients: $85 \%$ short chain peptides from hydrolysed whey protein, $15 \%$ free amino acids for optimal residue, low fat content with $47 \%$ of fat content from medium chain triglycerides, fiber free) were administrated at a rate of $20 \mathrm{ml} / \mathrm{h}$. On postoperative day 2, 1000 $\mathrm{ml}$ of tea and $1000 \mathrm{ml}$ of Nutrison Advanced Peptisorb at a rate of $20 \mathrm{ml} / \mathrm{h}$ were administered by continuous infusion via automated pump and on next postoperative days the rate increased to $10-15 \mathrm{ml}$ every 8 10 hours up to the target rate of $80 \mathrm{ml} / \mathrm{h}$. Since the patient didn't present abdominal discomfort, he received Nutrison Advanced Peptisorb at a rate of $80 \mathrm{~mL} / \mathrm{h}$ without dilution.

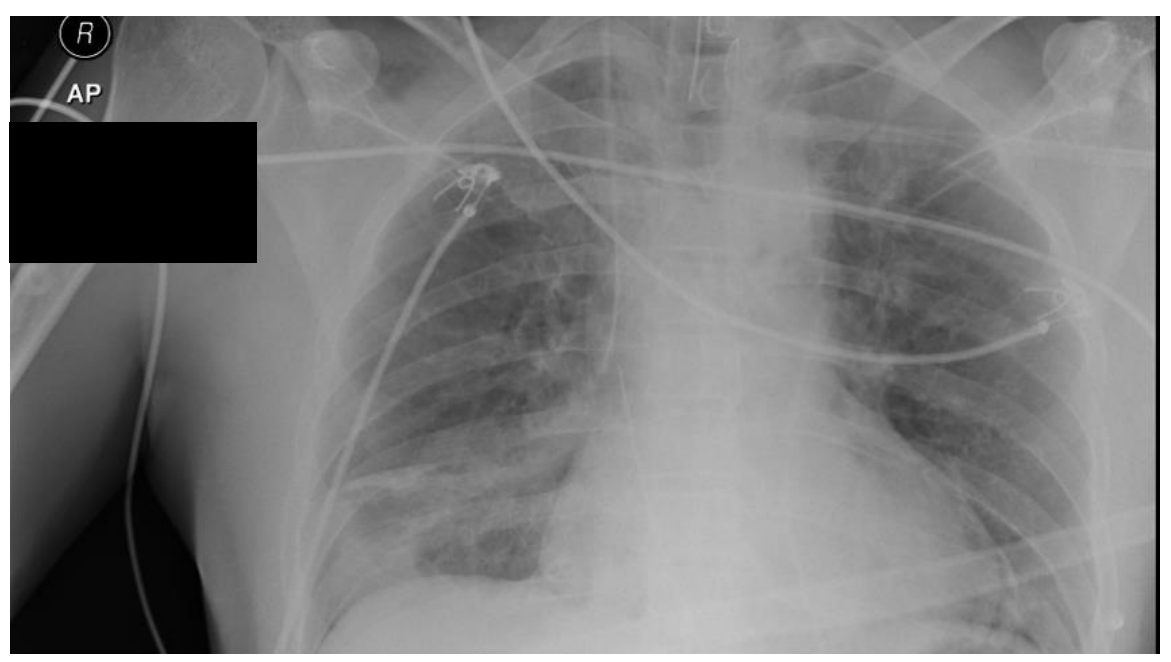

Fig. 7a. Plain chest X-ray immediately after the operation 


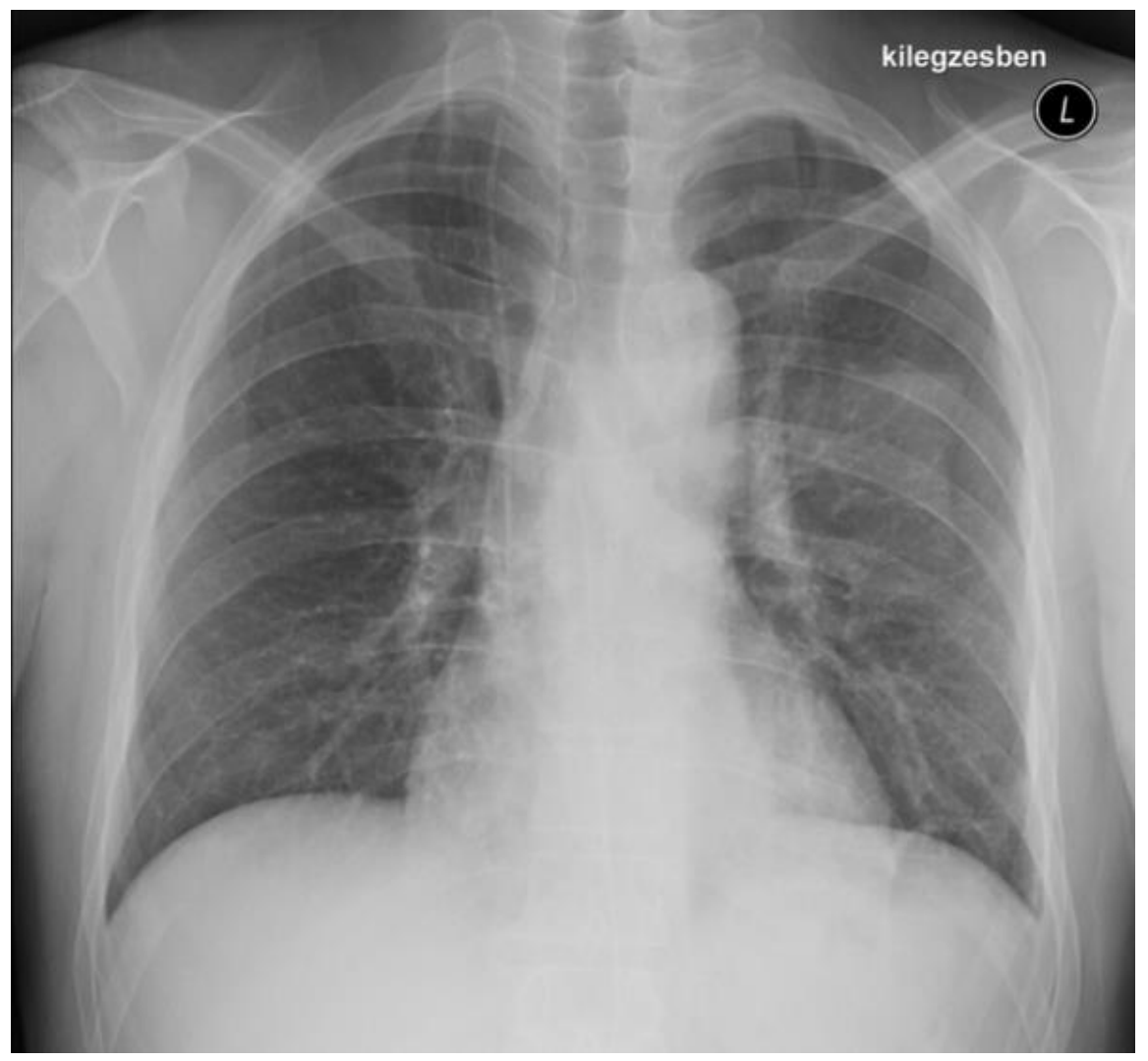

Fig. 7b. Plain chest x-ray (exhale) on discharge day

Table 2. Pre- and postoperative evolution of biochemical parameters

\begin{tabular}{lllll} 
Test Name & $\begin{array}{l}\text { Result-value on } \\
\text { admission }\end{array}$ & $\begin{array}{l}\text { Result value on } \\
\text { discharge }\end{array}$ & Unit & Normal value \\
\hline White Blood & 25.36 & 6.21 & Giga/L & $4-10$ \\
Cells & 90.4 & 63.4 & $\%$ & $45-70$ \\
L-NEU & 6.9 & 0.0 & $\%$ & $<5$ \\
Eosinophils & 0.2 & 0.6 & $\%$ & $<2$ \\
Basophils & 5.8 & 7.2 & $\%$ & $2-10$ \\
Monocytes & 3.6 & 21.9 & $\mathrm{Giga} / \mathrm{L}$ & $25-45$ \\
Lymphocytes & 22.9 & 3.93 & $\mathrm{~g} / \mathrm{L}$ & $1.8-7$ \\
NEUABS & 143 & 94 & $\mathrm{~L} / \mathrm{L}$ & $135-170$ \\
\hline Hemoglobin & 0.43 & 0.28 & $\mathrm{INR}$ & $0.39-0.52$ \\
Hematocrit & 1.05 & 1.08 & $\mathrm{umol} / \mathrm{L}$ & 1.1 \\
INR & 21.8 & 7.6 & $\mathrm{umol} / \mathrm{L}$ & 17 \\
\hline Total Bilirubin & 1.1 & $\mathrm{umol} / \mathrm{L}$ & $1.71-20.5$ \\
Direct Bilirubin & 37 & 43 & $\mathrm{U} / \mathrm{L}$ & $50-110$ \\
Creatinine & 81 & 24 & $\mathrm{U} / \mathrm{L}$ & $40-50$ \\
\hline TGO & 20 & 25 & $\mathrm{U} / \mathrm{L}$ & $40-50$ \\
\hline TGP & 16 & & $\mathrm{mmol} / \mathrm{L}$ & $50-70$ \\
\hline GGT & 14 & 3.7 & $\mathrm{ug} / \mathrm{L}$ & $3.5-5$ \\
\hline K & 3.2 & & $\mathrm{mg} / \mathrm{L}$ & $<0.5$ \\
\hline Procalcitonin & 0.54 & 23.5 & & $<5$ \\
\hline CRP & 27.3 & &
\end{tabular}

The patient was discharged 10 days after with normal analytical and clinical parameters and correct feeding via the jejunostomy.
Over the following period, the patient developed hypoalbuminemia due to poor enteral nutrition which was corrected in order 
to withstand the planned esophageal reconstruction operation.

In January 2018 cervical esophagogastrostomy (gastric pull-up) was performed to re-establish continuity of gastrointestinal tract. The postoperative period was uneventful.

On postoperative day 7, anastomotic integrity was confirmed with contrast imaging study (Figure 8) and the patient received clear fluids (oral administration) on postoperative day 8.

Enteral tube feeding was decreased and the diet was changed to full fluid consistency and was discontinued before the patient's discharge.

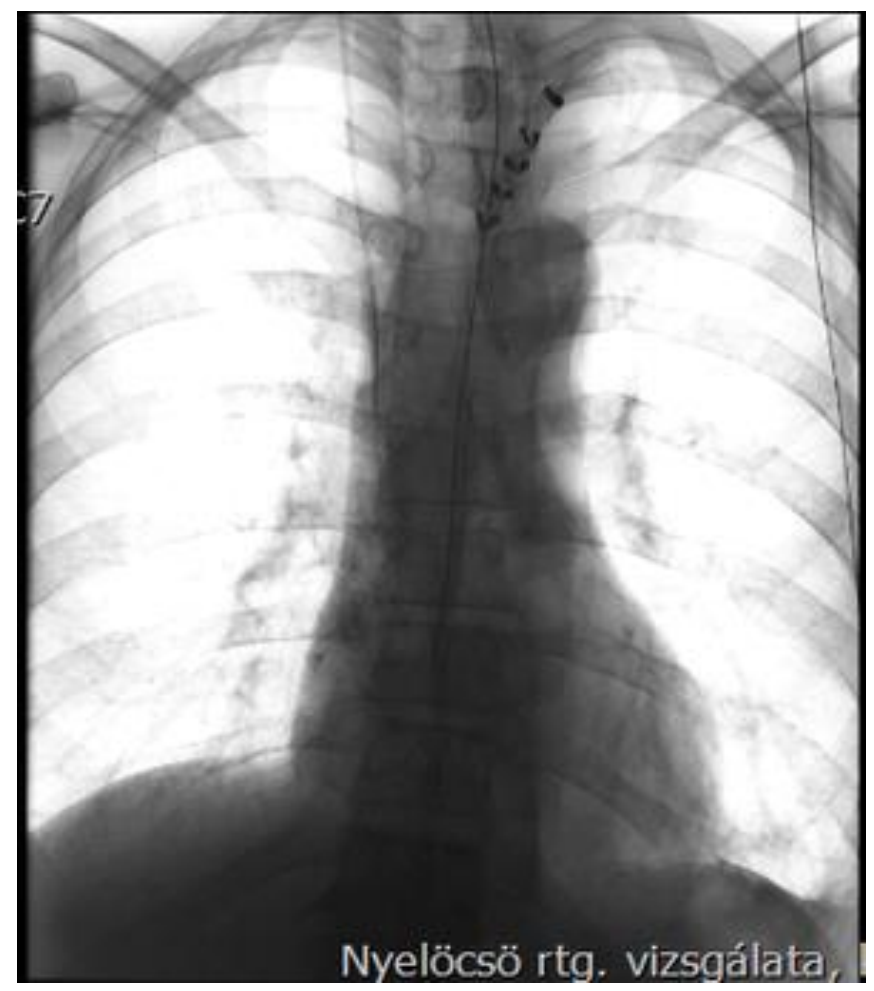

Fig. 8. Contrast imaging study confirming the integrity of the anastomosis

The patient was discharged after 12 days in a good general condition, physiological parameters, and showing a correct oral tolerance. After 1 year of follow-up, the patient maintains correct oral nutrition with no episodes of swallowing disorders, dysphagia, EFBI or malabsorption syndrome.

\section{Discussions}

EoE is a chronic, antigen-mediated inflammation of the esophagus in which oral and / or aeroantigens cause infiltration of the esophagus with eosinophil granulocytes, mucosal hyperplasia, and fibrosis of the subepithelial layers. It can occur in both children and adults, with two-thirds male predominance $[1,2]$.
Clinical symptoms are age-related, nutrition difficulties, growth retardation, GERDlike symptoms in children, and periodic dysphagia and EFBI in adults being the most common symptoms [3].

The main diagnostic tools of EoE are endoscopy and histological examination. It is recommended that serum $\mathrm{IgE}$, skin-Prick and Patch tests to be performed on nutrients and aeroantigens for simultaneous food and aeroallergies testing [3, 4].

EoE is a chronic disease requiring lifelong treatment. Therapy may include antiinflammatory medication: PPI therapy, systemic or topical corticosteroid treatment (budesonide, fluticasone). Alternatives to medication include diets based on the removal of trigger antigens from the diet. In the case of ineffectiveness of the first line treatments, the formed constrictions are expanded 
mechanically by means of an endoscopic balloon, the common complication of which is esophageal perforation [3-5]. Studies have shown that $31 \%$ of EoE patients have complications, from which $19 \%$ present vertical mucosal lacerations, $8 \%$ esophageal perforation with pneumomediastinum, and only $3 \%$ Boerhaave's syndrome $[10,11]$.

Differential diagnosis of EoE and GERD, achalasia, Crohn disease, hypereosinophilic syndrome, pill esophagitis or infectious esophagitis is important [12].

We presented the case of a young male patient who initially presented swallowing difficulties (which haven't been remitted with PPI therapy) and afterwards dysphagia and EFBI. These general symptoms can be caused by many different disorders. Initially we considered GERD but the patient did not complain about acid or nonacid reflux events, and PPI therapy did not remit the swallowing difficulties while the endoscopy did not show erosive esophagitis aspect. Secondly, the severe dysphagia determined us to study the possibility of achalasia, but plain chest radiography with a water soluble contrast swallow did not display the classic image of achalasia (bird beak-like appearance, incomplete relaxation of the lower esophageal sphincter). Our patient presented a mild eosinophilia and we have debated the possibility of hypereosinophilic syndrome, but blood tests have never shown more than $1.5 \mathrm{x}$ $10^{9}$ eosinophils/L and our investigations have not confirmed the evidence of eosinophil infiltration of other organs. Last, but not least, due to elevated inflammatory markers we have reflected on infectious esophagitis, but our patient did not presented any inflammatory symptoms until the esophagus perforation occurred and lead to mediastinitis.

Due to the fact that our patient's esophagus walls were more friable because of the tissue structure changes determinate by the EoE, perforation complicated the gastroscopy.

The particularity of our case consists in the unspecific clinical presentation and the noncompliant patient, which failed to warrant a complete and correct diagnosis and ultimately lead to invasive treatment and loss of the esophagus. In fact, EoE often begins with esophageal perforation determined by EFBI [2, 5].

Guidelines has shown that esophagus perforation can be treated non-surgically (conservative) or surgically. The conservative treatment is applied if the injury consists in cervical esophagus rupture and/or intramural perforations.

This statement is supported by another case report from our clinic (unpublished data): young male patient with foreign body (food supplement tablets) impaction in cervical esophagus; the tablet was not removed by gastroscopy; the plain chest radiography with a water soluble contrast swallow confirmed the complete obstruction and the intramural perforation of the esophagus; due the patient's good general condition and the nearly normal inflammatory parameters, we have abandoned the urgent surgical intervention and the gastroscopy were not necessary; Salem sump tube was placed into the esophagus and the patient was fed parenterally; on the third day of the observation, the tablet went was spontaneously rejected into the stomach; control gastroscopy and histological examination confirmed EoE diagnosis.

On the other hand, if the diagnose lasts less than 24 hours, the perforation affects only the thoracic or abdominal esophagus and if the esophageal rupture is complete, primer suture and mediastinum or thoracic drainage is performed. In case of delayed diagnose (>24 hours) or if other complications are present, esophageal resection and reconstruction is applied required [5, 13].

Esophageal perforation has a good prognosis if the patient is diagnosed early. If the treatment is prompt and well established correct (within 24 hours), the survival rate is over $90 \%$. If the treatment is delayed, the survival percentage drops to $50 \%$ [14]. Beside this, the mortality caused by esophageal perforation varies with etiology and perforation location. The highest rates are attributed to Boerhaave syndrome - up to $72 \%$, partly due to the difficulty in diagnosis, followed by iatrogenic - $19 \%$ and traumatic perforation $7 \%$ [15]. Cervical perforation has a low mortality compared to abdominal or thoracic perforation. Mortality and morbidity in esophageal perforation is most often due to an 
inflammatory response to the gastric contents of the mediastinum, pleural space and adjacent tissues, and the spread of infection to paraesophageal structures. Negative intrathoracic pressure may attract esophageal contents exacerbating the lesion.

Of the above mentioned options, in our case, conservative therapy was not possible due to advanced mediastinitis, consequent septic shock, stricture and large perforation. Therefore, we decided to urgently perform a transhiatal esophagectomy without thoracotomy and cervical esophagostomy with pyloromyotomy and feeding jejunostomy, although this operation placed a great burden on the patient. After six months of rehabilitation, we proceeded to reconstructive surgery also.

\section{Conclusions}

Unfortunately, EoE is difficult to diagnose due to its non-specific or GERD-like clinical symptoms and often leads to life threatening misdiagnosis.

\section{References}

1. Philpott $H$, Nandurkar $S$, Thien F, Gibson PR, Royce SG. Eosinophilic esophagitis: a clinicopathological review. Pharmacol Ther 2015; 146:12-22.

2. Noel RJ, Putnam PE, Rothenberg ME. Eosinophilic esophagitis. N Engl J Med 2004; 351(9):940-941.

3. Reed C, Woosley JT, Dellon ES. Clinical characteristics, treatment outcomes, and resource utilization in children and adults with eosinophilic gastroenteritis. Dig Liver Dis 2015; 47(3):197-201.

4. Abassa KK, Lin XY, Xuan JY, Zhou HX, Guo $Y W$. Diagnosis of eosinophilic gastroenteritis is easily missed. World J Gastroenterol 2017; 23(19):3556-3564.

5. Muir AD, White J, McGuigan JA, McManus KG, Graham AN. Treatment and outcomes of oesophageal perforation in a tertiary referral centre. Eur J Cardiothorac Surg 2003; 23(5):799-804.

6. Orringen MB, Sloan $\mathrm{H}$. Esophagectomy without thoracotomy. J Thorac Cardiovasc Surg 1978; 76(5):643-654.
In order to avoid these unpleasant events, it is important to consider EoE in every case of a young male patient who has swallowing complaints, dysphagia or EFBI. At the same time, we must keep in our mind other pathologies entities with similar symptoms: GERD, achalasia, Crohn's disease, connective tissue disorders, hypereosinophilic syndrome, pill esophagitis and infectious esophagitis.

\section{Consent}

Written informed consent was obtained from the patient for publication of this case report and accompanying images. A copy of the written consent is available for review by the Editor-in-Chief of this journal.

\section{Competing interests}

The authors declare that they have no competing interests. Authors have not received any financing for this publication, not having any other financial connections, direct or indirect, with any other organization(s).
7. Transhiatal Esophagectomy Information Page [https://www.ctsnet.org/article/transhiatalesophagectomy available at 06/25/2019]

8. Srinathan SK, Hamin $T$, Walter $S$, Tan $A L$, Unruh HW, Guyatt G. Jejunostomy tube feeding in patients undergoing esophagectomy. Can J Surg 2013; 56(6):409-414.

9. Nutrison Advanced Peptisorb Information Page [http://www.nutriciamedical.co.nz/media/catalog /product/n/u/nutrison_advanced_peptisorb_dec 2016.pdf available at 6/25/2019]

10. Cohen MS, Kaufman AB, Palazzo JP, Nevin D, Dimarino AJ Jr, Cohen S. An audit of endoscopic complications in adult eosinophilic esophagitis. Clin Gastroenterol Hepatol 2007; 5(10):1149-1153.

11. Vernon N, Mohananey D, Ghetmiri E, Ghaffari G. Esophageal rupture as a primary manifestation in eosinophilic esophagitis. Case Rep Med 2014; 2014:673189.

12. Eosinophilic esophagitis - Differential Diagnosis Information Page [https://online.epocrates.com/u/29351304/Eosi nophilic+esophagitis/Diagnosis/Differential available at 6/25/2019] 
13. Port JL, Kent MS, Korst RJ, Bacchetta M, Altorki NK. Thoracic esophageal perforations: a decade of experience. Ann Thorac Surg 2003; 75(4):1071-1074.

14. Mueller DK, Mancini MC, Govil $Y$, et al.: Esophageal Rupture Treatment \& Management [https://emedicine.medscape.com/article/42541 0-overview\#a5 available at 6/25/2019]

15. Zimmermann $M$, Hoffmann $M$, Jungbluth $T$, Bruch HP, Keck T, Schloericke E. Predictors of morbidity and mortality in esophageal perforation: retrospective study of 80 patients. Scan J Surg 2017; 106(2):126-132. 